\title{
Sugar and Dental Caries: Guidelines for Sugar Consumption Suggested by the World Health Organization
}

\author{
Dalila S Marques, Stella MF Lima* and Tatiana DPL Azevedo \\ Dentistry Course, Catholic University of Brasilia, Brazil \\ *Corresponding author: Stella MF Lima, Dentistry Course, Catholic University of Brasilia, Brazil
}

\begin{abstract}
Dental caries is a dynamic multifactorial disease, determined by sugar consumption and mediated by biofilm formation and activity that results in an imbalance between the processes of demineralization and remineralization of enamel. Untreated dental caries is the most prevalent oral diseases in the world and its treatment is one of the most expensive treatment. For these reasons, caries prevention must be mainly related to the control of sugar intake. Since 2002, some publications of WHO recommend the consumption of free sugars should be below $10 \%$ of the daily energy consumption. In 2015 , they published a new guide for adults and children, with a strong recommendation that the consumption of these sugars should be reduced in all life cycles. In 2019 , WHO published the "WHO Implementation Manual: Ending childhood dental caries", part of the "Oral Health Program, Prevention of Noncommunicable Diseases". This review aims to discuss the current guideline recommended by the World Health Organization for the consumption of sugar as an improvement approach for the prevention of dental caries.
\end{abstract}

Abbreviations: WHO: World Health Organization; GRADE: Grading of Recommendations Assessment Development and Evaluation; ECC: Early Childhood Caries

\section{Introduction}

The Global Burden of Disease Study 2017 revealed that 3.5 billion people worldwide are affected by oral diseases. Untreated dental caries is the most prevalent non-communicable oral diseases [1]. Despite the advances in preventive actions, caries is still a prevalent disease, causing pain, anxiety, and limitations in daily activities. Indeed, caries development can directly affect the population's quality of life. The treatment for caries is the 4th most expensive treatment among diseases [2]. Dental caries is a dynamic multifactorial disease, determined by sugar consumption and mediated by biofilm formation and activity that results in an imbalance between the processes of demineralization and remineralization of enamel. It is determined by biological, behavioral, psychosocial factors, and also the individual's environment [3]. In 1989, for the first time, the World Health Organization (WHO) established dietary guidelines for controlling the consumption of free sugars. Afterward, several documents were produced, and, in 2015, after rigorous analysis of scientific articles, the "Guideline: Sugars Intake for Adults and Children" was published. This important guideline recommends the adequate consumption of free sugars to reduce the prevalence of non-communicable diseases in adults and children, focused on the prevention and control of obesity and dental caries [4]. This review aims to discuss the current guideline recommended by the World Health Organization for the consumption of sugar as an improvement approach for the prevention of dental caries.

\section{Literature Review}

Dental caries is considered a dynamic multifactorial disease and is currently considered a non-communicable disease and shares risk factors common to other non-communicable diseases associated with sugar consumption, such as cardiovascular disease, diabetes, and obesity [3]. For these reasons, caries prevention must be mainly related to the control of sugar intake. Sugars are classified into monosaccharides (glucose, galactose, and fructose) and disaccharides (sucrose, maltose, and lactose). Sugars located in the cellular structure of grains, fruits, and vegetables and those naturally present in milk and its derivatives are considered natural sugars. These sugars may not significantly contribute to the development of caries due to the amount of fiber and water [4]. It also possesses protective factors such as polyphenolic compounds and calcium. Hence, the impact of fruits, vegetables, and grains on the mechanical stimulation of salivary flow, would help to reduce the risk of caries related to its consumption [5]. Other sugars that are not considered to be natural are classified by the WHO as 
free sugars. All monosaccharides and disaccharides are added to food during preparation and can be processed or not. The sugars naturally present in honey, syrups and fruit juices are also classified as free sugars. Accordingly, the consumption of these foods should be controlled to prevent non-communicable diseases better [5].

Since 2002, some publications of WHO recommend the consumption of free sugars should be below $10 \%$ of the daily energy consumption [6]. In 2015, they published a new guide for adults and children, with a strong recommendation that the consumption of these sugars should be reduced in all life cycles. It was emphasized the need to control sugar consumption to below $10 \%$, also providing an additional recommendation for a subsequent reduction to 5\% [4]. Adults with a healthy body mass index could consume 50 grams of free sugars (about ten teaspoons of sugar), considering $10 \%$ of their daily energy consumption. These sugars are present in sweets, soft drinks, pasta, stuffed cookies, cakes, among others. The amount of free sugars in some foods is shown in Table 1. The recommendations of the WHO guidelines were related to the Grading of Recommendations Assessment Development and Evaluation (GRADE) process [7]. Therefore, the WHO guidelines were based on significant evidence according to the type of study, limitations, consistency, presence of bias, effect size, dose-response, and analysis of confounding variables. After considering these factors, the evidence could be classified as high, moderate, low, or very low.

Table 1: Determination of the amount of sugar per serving indicated in some foods consumed by children.

\begin{tabular}{|c|c|c|}
\hline Food & Volume/Weight & Amount of sugar \\
\hline Milk & $200 \mathrm{~mL}(1 \mathrm{cup})$ & 9 grams \\
\hline Soda & $200 \mathrm{~mL}(1 \mathrm{cup})$ & $15-20$ grams \\
\hline Artificial juice & $200 \mathrm{~mL}(1 \mathrm{cup})$ & 18 grams \\
\hline Chocolate powder & $\begin{array}{c}20 \text { grams }(2 \\
\text { tablespoons })\end{array}$ & $15-18$ grams \\
\hline Cookie & 30 gramas $(3$ units $)$ & $6-12$ grams \\
\hline Chocolate & 30 grams & $12-18$ grams \\
\hline Tomato sauces & 8 grams & 2 gramas \\
\hline Mayonnaise & 12 grams & 0.3 gram \\
\hline Corn flakes & 30 grams & $9-12$ grams \\
\hline $\begin{array}{c}\text { Papaya and orange } \\
\text { baby food }\end{array}$ & 120 grams & 11 grams \\
\hline
\end{tabular}

The analysis of cohort studies in children suggested a positive association between the number of free sugars ingested and dental caries. The evidence suggested that when the intake of these foods was higher than $10 \%$ of daily consumption, the experience with caries was also more significant. In addition, epidemiological studies demonstrated a low prevalence of caries in individuals with low consumption of sugar per year (less than $10 \mathrm{~kg}$, approximately $5 \%$ of the total energy consumption). Overall, there is a doseresponse between the number of free sugars ingested and the prevalence of caries. The GRADE process classified these evidences as moderate significance [8]. Conceivably, the WHO produced recommendations for the consumption of sugar by adults and children [4]. The frequency of sugar ingestion and the amount of sugar consumption can be considered risk factors for tooth decay. Although few epidemiological assess this relationship, the WHO concluded that both variables are essential [4]. The WHO meeting Expert Consultation on Public Health Intervention against Early Childhood Caries (ECC) in Bangkok, Thailand, in 2016 discussed several strategies to prevent caries in the first years of life. The need to control sugar consumption has been reinforced so that it does not exceed 10\% [3]. In 2019, WHO published the "WHO Implementation Manual: Ending childhood dental caries", part of the "Oral Health Program, Prevention of Non-communicable Diseases" [9]. This manual discusses nutritional recommendations, encouraging exclusive breastfeeding until six months of age, and avoiding the consumption of free sugars in drinks and food in the first two years of life. The WHO also recommends that governments publish national guidelines on food and nutrition. Brazil developed food guides according to the cultures of the country and its populations.

In 2002, the first version of the Food Guide was published and then revised in 2010 [10,11]. This publication demonstrated the 10 Steps to Healthy Eating. Later, a new edition was prepared in 2019 due to social changes and changes in eating in recent years [12]. This document also recommended food and nutrition education actions on an individual and collective level in the Unified Health System (SUS) and in other sectors. This is a guide for policies, programs and, actions that aim to support, protect, and promote the health and food and nutritional security of Brazilian children. In this updated version, "12 Steps for Healthy Eating" were suggested. In this regard, the impact of the steps to healthy eating was evaluated according to a randomized clinical trial. Individuals that followed the dietary guidelines proposed by the guide demonstrated a higher proportion of children breastfed exclusively until six months of age, and they experience fewer events of caries [13].

\section{Conclusions}

Dental caries is one of the most prevalent non-communicable disease in the world. Despite the use of fluoridated toothpaste, caries is a multifactorial disease, and all contributing factors must be known and controlled. Preventive measures, as dietary guidance, has been discussed over the years. The patient's eating habits must be registered by the dentist to identify the frequency and amount of ingestion of free sugars. One way to conduct this interview is through the 24-hour Recall Diary, a method in which the patient informs everything that was ingested the previous day. The WHO recommendation for free sugars consumption $(10 \%$ of the total daily intake) [4] is an alert for the worldwide population, since many processed or ultra-processed foods, can carry this amount of sugar in a single portion (Table 1). The WHO guidelines reinforce the need for this control to be carried out in all life cycles since caries is a cumulative disease [4]. In addition, these recommendations are useful in guiding public policies and in managing health promotion programs, such as restricting the amount of sugar for foods, emphasizing the warning of food composition in its labels, and encouraging the eating of healthy food. In this way, all efforts 
should be directed towards the prevention of caries once it is a prevalent disease-causing pain, discomfort, limitations, anxiety, in addition to impairing the child's school development, social life, and, consequently, their quality of life.

\section{References}

1. Marcenes W, Kassebaum NJ, Bernabe E, Flaxman A, Naghavi M, et al. (2013) Global burden of oral conditions in 1990-2010: a systematic analysis. J Dent Res 92(7): 592-597.

2. Petersen PE, Bourgeois D, Ogawa H, Estupinan Day S, Ndiaye C (2005) The global burden of oral diseases and risks to oral health. Bull World Health Organ 83(9): 661-669.

3. Pitts N, Baez R, DiazGuallory C (2019) Early Childhood Caries: IAPD Bangkok Declaration. Int J Paediatr Dent 29(3): 384-386.

4. (2015) World Health Organization. Sugars intake for adults and children. Geneva.

5. Moynihan P (2016) Sugars and Dental Caries: Evidence for Setting a Recommended Threshold for Intake. Adv Nutr 7(1): 149-156.

6. (2003) World Health Organization. Diet, nutrition and the prevention of chronic disease. Word Health Organization Technical Report 91(6v-6viii): 1-149.
7. Atkins D, Best D, Briss PA, Eccles M, Falck Ytter Y, et al (2004) Grading quality of evidence and strength of recommendations. BMJ 328(7454): 1490.

8. Moynihan PJ, Kelly SA (2014) Effect on caries of restricting sugars intake: systematic review to inform WHO guidelines. J Dent Res 93(1): 8-18.

9. (2019) World Health Organization. Ending childhood dental caries: WHO implementation manual.

10. (2002) Ministry of Health. Ten steps to healthy eating: food guide for children under two years old. Brasília: Ministry of Health / Pan American Health Organization, Brazil.

11. (2010) Ministry of Health. Ten steps to healthy eating: food guide for children under two years. Brasília: Ministry of Health / Pan American Health Organization, Brazil.

12. (2019) Ministry of Health. Food guide for Brazilian children under two years. Ministry of Health, Brazil.

13. Vitolo MR, Borlolini GA, Feldens CA (2005) Impacts of the implementation of the ten steps of healthy eating for children: randomized field trial. Cad. Public Health 21(4): 1448-1457.

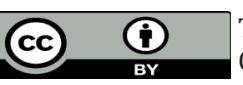

This work is licensed under Creative Commons Attribution 4.0 License

To Submit Your Article Click Here: Submit Article

DOI: $10.32474 /$ IPDOAJ.2020.04.000176

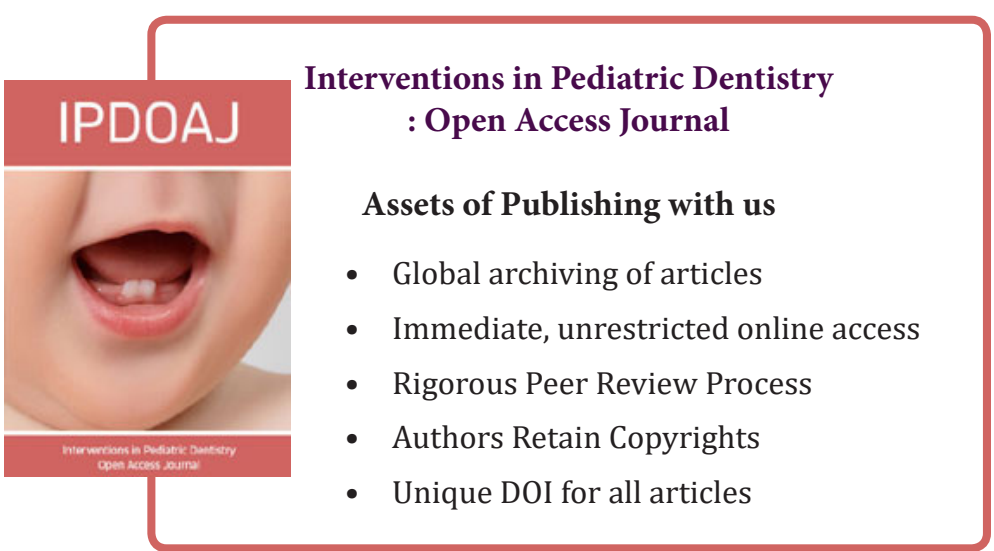

